\title{
Semicontinous Versus Interrupted Suture Technique For Mitral Valve Replacement In Patient With Rheumatic Mitral Valve Disease
}

\section{Shimaa A.Mosallom ${ }^{\text {a }}$, Karam M.Eisa ${ }^{\text {a }}$, Mohamed Negm ${ }^{\text {, }}$, Mohamed Abdel-Bary ${ }^{a}$}

\author{
${ }^{a}$ Department of Cardiothoracic Surgery, Faculty of Medicine, South valley UniversityUniversity, Qena, Egypt. \\ ${ }^{b}$ Department of General Surgery, Faculty of Medicine, South valley University University, Qena, Egypt.
}

\section{ABSTRACT:}

Background: In mitral valve prolapse is associated with severe mitral regurgitation, mitral valve repair or surgical replacement may be necessary. In Mitral valve replacement, some surgeon use continuous suture, others use interrupted suture technique.

Aim of the work: to compare efficacy of semi-continuous technique \& interrupted suture of prosthestic valve replacement in mitral valve replacement.

Patients and Methods: it was a prospective randomized comparative study done at the Cardiothoracic Surgery Department, Qena University Hospitals, South Valley University, Egypt between April 2016 to April 2018. It included 31 patients with rheumatic mitral valve disease whatever the cause. They were divided into group1: patients underwent semi-continuous technique for MVR (18 cases) and group2: patients underwent interrupted technique (13 cases).All of patients were subjected to preoperative data: complete history, general examination including general condition of the patient and vital signs, investigations including routine laboratory investigations, especially complete blood count, chest X ray (CXR), 12 lead Electrocardiography (ECG), echocardiography and coronary angiography if patient more than 40 years old, operative data including total bypass time, aortic CCT, number of sutures, and postoperative outcome including ICU stage and inotrope use, postoperative complications, pulmonary hypertension and echo findings as immediate postoperative and echo after 1 and 3 months postoperative.

Results: We found that the mean total bypass time was significantly higher among interrupted group compared to semicontinuous one.

Conclusion: Semicontinuous sutures showed significantly better results than interrupted ones, especially regarding the total bypass time

Keywords: mitral valve replacement, Semicontinuous, interrupted, Suture, rheumatic.

\section{Introduction:}

Mitral stenosis is a valvular heart disease (most common of rheumatic heart disease) characterized by the narrowing of the orifice of the mitral valve of the heart. When the mitral valve area goes less than $1 \mathrm{~cm}^{2}$, there will be an increase in the left atrial pressures. Since the normal left ventricular diastolic pressures is about $5 \mathrm{mmHg}$, a pressure gradient across the mitral valve of $20 \mathrm{mmHg}$ due to severe mitral stenosis will cause a left atrial pressure of about $25 \mathrm{mmHg}$. This left atrial pressure is transmitted to the pulmonary vasculature and causes pulmonary hypertension(Carabello 2005).
Mitral valve prolapse is a condition in which the two valve flaps of the mitral valve do not close smoothly or evenly, but instead bulge (prolapse) upward into the left atrium. In mitral valve prolapse is associated with severe mitral regurgitation, mitral valve repair or surgical replacement may be necessary(Honda et al., 2016).

In Mitral valve replacement, some surgeon use continuous suture, others use interrupted suture technique. The continuous suture technique has numerous advantages as a simple, quick, and effective method for valve replacement. The semicontinuous suture technique is a modification of 
the continuous technique aiming to avoid its technical disadvantages. This method combines advantages of the continuous and interrupted suture techniques. The semi-continuous suture technique is suitable for all types of prosthetic valve replacement, especially those of rheumatic origin, and with small left atrium and small aortic annulus. It is simple, with short period of valve implantation, associated with few postoperative complications, and especially suitable for patients in developing countries(Honda et al., 2016).

\section{Aim of the work:}

1. Compare efficacy of semi continuous technique \&interrupted suture of prosthestic valve replacement.

2. Describe advantage of semicontinous suture and describe the most frequent errors and important details about disadvantage and complication of interrupted suture of prosthestic valve replacement.

\section{Patients and Methods:}

Design: it was prospective randomized comparative study done at the Cardiothoracic Surgery Department, Qena University Hospitals, South Valley University, Egypt between April 2016 to April 2018.

Patients: our study included 31 patients with rheumatic mitral valve disease whatever the cause. They were divided into

- Group1: Patients underwent semi-continuous technique for MVR (18 cases).

- Group2: Patients underwent interrupted technique (13 cases).

Both groups were matched as regards age, sex and preoperative cardiac data (according to preoperative echo) with no significant differences.

\section{Inclusion criteria:}

- All patients presented with rheumatic mitral valve disease whatever the cause

- Agreed to be included in the research and to sign the informed written consent.

\section{Exclusion criteria:}

- Ischemic mitral regurge.

- Any cause other than mitral valve disease.

- Patient refusal.

- Patients with incomplete data.

- Patients with multiple risk factor.

- Patient Redo, CABG, AVR.

Methods: All of patients were subjected to the following:
A) Preoperative data:

I.Full complete history: Personal and demographic data: name, age, sex, socioeconomic status, etc, symptoms as Fatigue, exercise intolerance, dyspnea, chest pain, paroxysmal nocturnal dyspnea, heart failure, comorbidities: Diabetes, hypertension or tobacco use, smoking and history of medications: anti-coagulants and antihypertensive.

II. General examination including general condition of the patient and vital signs.

III. Investigations including outine laboratory investigations, especially complete blood count, chest $\mathrm{X}$ ray (CXR), 12 lead Electrocardiography (ECG), echocardiography and coronary angiography if patient more than 40 years old.

B) Operative data including total bypass time, aortic CCT, number of sutures.

C)Postoperative outcome including

I. Immediate postoperative data as ICU stage and inotrope use.

II. Postoperative complications.

III. Postoperative pulmonary hypertension.

IV. Postoperative echo findings as immediate postoperative and echo after 1 and 3 months postoperative.

\section{Statistical analysis:}

- Statistical package for social sciences (IBMSPSS), version 25 IBM- Chicago, USA (May 2017) was used for statistical data analysis.

- Data expressed as mean, standard deviation (SD), number and percentage. Mean and standard deviation were used as descriptive value for quantitative data, while number and percentage were used to describe qualitative data.

- Student $t$ test was used to compare the means between two groups, and one-way analysis of variance (ANOVA) test was used to compare means of more than two groups. Mann Whitney test was used instead of Student $t$ test in case of non parametric data.

- Pearson Chi square was used to compare percentages of qualitative data, and Fisher's Exact test was used for non parametric data.

- For all these tests, the level of significance (Pvalue) can be explained as:
$\circ$ No significance $P>0.05$
$\circ$ Significance $\mathrm{P}<0.05$ 
○ High significance $\mathrm{P}<0.001$.

\section{Results:}

Our study population included 31 patients presenting with rheumatic heart with mitral stenosis disease, divided into 2 groups, one group was subjected to semi-continuous suturing (18 cases; $58.1 \%$ ) and the other group was subjected to interrupted suturing (13 cases; $41.9 \%$ ).

Regarding the age of the study group, we observed that the mean age of our study patients was around 40 years, with no significant difference between the two groups $(\mathrm{p}$ value $=0.640(\mathrm{NS})$.

Regarding the sex of the study group, we observed that around two thirds of our cases were females; and the two groups were sex matched, with no significant differences between them ( $\mathrm{p}$ value $=$ 0.718 (NS). Against to our study, Azam et al. 2015 found that most of the studied group were male (74) but, with non-significant difference between both groups.

We found that preoperative cardiac data were similar between the two groups, with no significant differences. The majority of cases had sinus rhythm, with AF seen in 12 cases ( 8 from semicontinuous group and 4 from $\mathrm{AF}$ group). Regarding risk factors, the majority of cases had no detectable risk factor, while 13 cases had hypertension, either alone (in 11 cases) or combined with DM (2 cases). NYHA class showed that only a minority of cases had NYHA class II, with over $60 \%$ had NYHA class III and nearly one quarter had class IV. Most of the cases had preoperative complications, with arrhythmias were the most common (seen in 11 cases); followed by palpitation (10 cases) and stroke (6 cases).

Regarding the preoperative ECHO data (table 1), we found that all of the preoperative Echo measures were similar between the two groups, with no significant differences between them. The only exception was $\mathrm{PH}$ which was significantly higher (50.38) among semi-continuous group, compared to interrupted one (only 38), with a $\mathrm{p}$ value of 0.04 .

Regarding the intraoperative data (table 2), we found that the mean total bypass time was around 100 minutes among all cases, with slightly higher duration among interrupted group (105 minutes) compared to semi-continuous one (98 minutes) but with no significant difference (P value: 0.169 (NS). Also; aortic CCT was slightly higher among interrupted group compared to semicontinous one, with non-significant difference (P value: 0.386 (NS).

On the other hand, the number of sutures was largely higher among interrupted group (12.2) compared to semi-continuous one (4); with highly significant difference (P value: $<0.001$ (HS).

Regarding the immediate postoperative data, we found that the ICU stage immediate postoperatively was significantly higher among interrupted group (6.8) compared to semicontinuous one (4.5). Regarding inotrope use, the only significant one was dobutamine (in over half of interrupted cases; $54 \%$, compared to only $17 \%$ among semi-continuous one, $p$ value 0.036 ). Although adrenaline was much higher among semi-continuous group (50\% compared to $23 \%$ among interrupted group); the difference was non significant. GTN was non significantly higher among interrupted group (100\%) compared to semi-continuous one (89\%).

Regarding the postoperative complications, we found that postoperative complications were significantly higher among interrupted group (54\%) compared to only 2 cases (11\%) among semi-continuous one ( $\mathrm{p}$ value $=0.046)$.

As regards the Pulmonary blood pressure immediate postoperatively, we found that immediate postoperative pulmonary blood pressure was slightly higher among semi-continuous group (40 $\mathrm{mmHg}$ ) compared to interrupted one (38.6 $\mathrm{mmHg}$ ); with non-significant difference ( $\mathrm{P}$ value $=$ 0.740 (NS).

Regarding the immediate postoperative Echo findings, we found that all of the immediate postoperative Echo measures were similar between the two groups, with non-significant differences.

Regarding the immediate postoperative Echo valve findings, we found that all of the cases showed immediate postoperative normally functioning valves, with the exception of trivial PV seen in only 2 of the semicontinous cases; with nonsignificant difference between the two groups.

Regarding the 1 month postoperative Echo data, we found that after one month of the operation, all of the Echo findings were similar between the two groups, with non significant differences between them.

Regarding the one month postoperative Echo valve findings, we found that after one month, valves were normally functioning among all cases, with the exception of trivial PV, mild PV and central 
MR; seen in one case of the interrupted group each; with non significant difference between the two groups.

Regarding the 3 month postoperative Echo data, we found that after 3 months of the operation, EF and LVPWT showed non significant differences between the two groups. The exception was PHT which was significantly higher among semicontinuous group (36.9) compared to the interrupted one (30.1); with a significant difference $(\mathrm{p}$ value $=0.033)$.

Regarding the three month postoperative Echo valve findings, we found that after three months, valves were normally functioning among all cases, with the exception of trivial PV, mild PV and moderate PV; seen in one case of the semicontinuous group each and mild central MR, seen in one case of the interrupted group; with nonsignificant difference between the two groups ( $p$ value $=0.292(\mathrm{NS})$.

Regarding the associated TR, we found that after three months, the majority of cases had either TR (seen in around $75 \%$ of cases); which was either mild or moderate in most of the cases, and severe only in 2 cases, with non significant difference between the two groups $(\mathrm{p}$ value $=0.322(\mathrm{NS})$ (table 3).

Table 1: Preoperative ECHO data

\begin{tabular}{llcccc}
\hline & Group & Mean & SD & T test & P value \\
LVEDD $(C M)$ & Semi-continuous & 5.224 & 0.717 & 0.489 & 0.629 \\
& Interrupted & 5.067 & 1.015 & & $(\mathrm{NS})$ \\
LVESD $(\mathrm{CM}$ & Semi-continuous & 3.435 & 0.545 & 0.833 & 0.796 \\
& Interrupted & 3.500 & 0.790 & & $(\mathrm{NS})$ \\
AO ROOT & Semi-continuous & 5.4612 & 0.849 & 1.743 & 0.093 \\
& Interrupted & 4.9583 & 0.624 & & $(\mathrm{NS})$ \\
RV & Semi-continuous & 3.019 & 0.429 & 0.695 & 0.493 \\
& Interrupted & 3.125 & 0.357 & & $(\mathrm{NS})$ \\
EF & Semi-continuous & 2.614 & 0.302 & 1.566 & 0.152 \\
& Interrupted & 3.025 & 0.585 & & $(\mathrm{NS})$ \\
FS & Semi-continuous & $58.75 \%$ & $8.06 \%$ & 0.168 & 0.868 \\
& Interrupted & $58.25 \%$ & $7.36 \%$ & & $(\mathrm{NS})$ \\
IVSD & Semi-continuous & $29.11 \%$ & $4.04 \%$ & 1.955 & 0.072 \\
& Interrupted & $33.53 \%$ & $4.66 \%$ & & $(\mathrm{NS})$ \\
LVPWD & Semi-continuous & 0.960 & 0.181 & 0.821 & 0.420 \\
& Interrupted & 0.909 & 0.114 & & $(\mathrm{NS})$ \\
MVR & Semi-continuous & 0.943 & 0.109 & 0.494 & 0.626 \\
& Interrupted & 0.967 & 0.137 & & $(\mathrm{NS})$ \\
PH & Semi-continuous & 1.318 & 0.733 & 1.064 & 0.297 \\
& Interrupted & 1.073 & 0.249 & & $(\mathrm{NS})$ \\
& Semi-continuous & 50.38 & 18.844 & $\mathbf{2 . 2 5 3}$ & $\mathbf{0 . 0 4 0}$ (S) \\
\hline
\end{tabular}

Table 2: intraoperative data.

\begin{tabular}{llcccc}
\hline & Group & Mean & SD & T test & P value \\
Total Bypass Time & Semi-continuous & 92.67 & 16.26 & $\mathbf{3 . 0 4 2}$ & $\mathbf{0 . 0 0 5}(\mathbf{S})$ \\
& Interrupted & 109.85 & 14.39 & & \\
Cross clamp time & Semi-continuous & 39.77 & 10.61 & $\mathbf{3 . 7 8 8}$ & $\mathbf{0 . 0 0 1}(\mathbf{S})$ \\
& Interrupted & 52.74 & 8.43 & & \\
Cardiopulmonary & Semi-continuous & 72.12 & 21.22 & $\mathbf{2 . 1 5 5}$ & $\mathbf{0 . 0 4 0}(\mathbf{S})$ \\
bypass time & Interrupted & 89.33 & 22.45 & & \\
Postoperative & Semi-continuous & 6.5 & 0.9 & 1.884 & $0.070(\mathrm{NS})$ \\
intubation time (hours) & Interrupted & 7.2 & 1.1 & & \\
Hospital stay (days) & Semi-continuous & 9.8 & 1.3 & 0.663 & $0.512(\mathrm{NS})$ \\
& Interrupted & 10.1 & 1.2 & & \\
\hline
\end{tabular}




\begin{tabular}{llcccc}
\hline Aortic CCT & Semi-continuous & 70.89 & 23.704 & 0.883 & 0.386 (NS) \\
& Interrupted & 76.23 & 8.348 & & \\
Number of suture & Semi-continuous & 4.00 & 0.000 & $\mathbf{6 7 . 6 7 3}$ & $<\mathbf{0 . 0 0 1}$ (HS) \\
& Interrupted & 12.23 & 0.439 & & \\
\hline
\end{tabular}

Table 3: associated TR.

\begin{tabular}{llccc}
\hline & & Group & Total \\
Associated TR & Semi-continuous & Interrupted & \\
& Non & $5(27.8 \%)$ & $3(23.1 \%)$ & $8(25.8 \%)$ \\
& Trace TR & 0 & $1(7.7 \%)$ & $1(3.2 \%)$ \\
& Mild TR & $10(55.6 \%)$ & $5(38.5 \%)$ & $15948.4 \%)$ \\
& Moderate TR & $3(16.7 \%)$ & $2(15.4 \%)$ & $5(16.1 \%)$ \\
& Severe TR & 0 & $2(15.4 \%)$ & $2(6.5 \%)$ \\
\hline
\end{tabular}

the pulmonary vasculature and causes pulmonary

\section{Discussion}

Heart valve replacement is a well-established and safe procedure with a low mortality risk, and results in considerable benefits to patients with chronic valvular disease. Mitral valve replacement (MVR) is performed using either semi-continuous (SC) technique or interrupted suture technique. The $\mathrm{SC}$ technique is quicker, with shorter aortic crossclamp and cardiopulmonary bypass times(Qicaiet al., 2006).

Incidence of paraprosthetic leakage (PPL) detected by trans-oesophageal echocardiograph (TEE) is about $15 \%$ after MVR in the immediate postoperative period (Ionescu et al., 2003) and 12.5\% without the use of TEE(Bonnefoy et al.,1995).

The degree of annular calcification, infection, type of suture technique, and size and type of prosthesis are considered to be the main contributing factors for PPL(Genoni et al., 2000).

It has been reported that the rate of PPL in SC method is high following the surgery compared to the interrupted suture technique. This leakage is not high in rheumatic valvular disease because of the thick and fibrotic annulus(Cooley 1984).

Mitral stenosis is a valvular heart disease (most common of rheumatic heart disease) characterized by the narrowing of the orifice of the mitral valve of the heart(Carabello 2005).

When the mitral valve area goes less than $1 \mathrm{~cm}^{2}$, there will be an increase in the left atrial pressures. Since the normal left ventricular diastolic pressures is about $5 \mathrm{mmHg}$, a pressure gradient across the mitral valve of $20 \mathrm{mmHg}$ due to severe mitral stenosis will cause a left atrial pressure of about 25 $\mathrm{mmHg}$. This left atrial pressure is transmitted to hypertension.

Mitral valve prolapse is a condition in which the two valve flaps of the mitral valve do not close smoothly or evenly, but instead bulge (prolapse) upward into the left atrium. In mitral valve prolapse is associated with severe mitral regurgitation, mitral valve repair or surgical replacement may be necessary.

In Mitral valve replacement,some surgeon use continuous suture, others use interrupted suture technique(Honda et al., 2016).

The continuous suture technique has numerous advantages as a simple, quick, and effective method for valve replacement. The semicontinuous suture technique is a modification of the continuous technique aiming to avoid its technical disadvantages. This method combines advantages of the continuous and interrupted suture techniques. we evaluated the semi-continuous suture technique in patients undergoing prosthetic valve replacement, comparing it with the conventional interrupted suture technique. in continuous suture. alsoparavalvular leakage was less in semi continuous suture,endocarditis\& valve thrombosis complication less in semi continuous technique

The semi-continuous suture technique is suitable for all types of prosthetic valve replacement, especially those of rheumatic origin, and with small left atrium and small aortic annulus. It is simple, with short period of valve implantation, associated with few postoperative complications, and especially suitable for patients in developing countries.

Our study was randomized prospective study, was conducted at cardiothoracic department at Qena Hospital University from May2017 to April 2018 
and included 60 patients presenting with rheumatic heart with mitral stenosis disease.

Our study population included 31 patients, divided into 2 groups, one group was subjected to semicontinuous suturing (18 cases; $58.1 \%$ ) and the other group was subjected to interrupted suturing (13 cases; $41.9 \%$ ). A study done by Azam et al. 2015 included 100 patients and divided into two equal groups of 50 to 50 each.

Regarding the age of the study group, we observed that the mean age of our study patients was around 40 years, with no significant difference between the two groups $(\mathrm{p}$ value $=0.640(\mathrm{NS})$. As found in (Azamet al., 2015).

Regarding the sex of the study group, we observed that around two thirds of our cases were females; and the two groups were sex matched, with no significant differences between them ( $\mathrm{p}$ value $=$ 0.718 (NS). Against to our study, Azam et al. 2015 found that most of the studied group were male (74) but, with non-significant difference between both groups.

We found that preoperative cardiac data were similar between the two groups, with no significant differences. The majority of cases had sinus rhythm, with AF seen in 12 cases ( 8 from semicontinuous group and 4 from AF group).

Regarding risk factors, the majority of cases had no detectable risk factor, while 13 cases had hypertension, either alone (in 11 cases) or combined with DM (2 cases). NYHA class showed that only a minority of cases had NYHA class II, with over $60 \%$ had NYHA class III and nearly one quarter had class IV. Most of the cases had preoperative complications, with arrhythmias were the most common (seen in 11 cases); followed by palpitation (10 cases) and stroke (6 cases).

Regarding the preoperative ECHO data, we found that all of the preoperative Echo measures were similar between the two groups, with no significant differences between them. The only exception was $\mathrm{PH}$ which was significantly higher (50.38) among semi-continuous group, compared to interrupted one (only 38 ), with a p value of 0.04 . Agree with our study, Azam et alfound that non-significant difference between both groups regarding the preoperative characteristics(Azamet al., 2015).

Regarding the intraoperative data, we found that the mean total bypass time, cross clamp time and cardiopulmonary bypass time all were significantly lower among semi-continuous group (93, 40 and 72 minutes; respectively) compared to the interrupted suture group (110, 53 and 89 minutes; respectively) with significant difference ( $\mathrm{P}$ values: $0.005,0.001$ and 0.040 respectively).

Also; aortic CCT was slightly higher among interrupted group compared to semi-continuous one, with non-significant difference ( $\mathrm{P}$ value: 0.386 .

On the other hand, the number of sutures was largely higher among interrupted group (12.2) compared to semi-continuous one (4); with highly significant difference (P value: $<0.001$ (HS).

Regarding the immediate postoperative data, we found that the ICU stage immediate postoperatively was significantly higher among interrupted group (6.8) compared to semicontinuous one (4.5). Regarding inotrope use, the only significant one was dobutamine (in over half of interrupted cases; $54 \%$, compared to only $17 \%$ among semi-continuous one, $\mathrm{p}$ value 0.036). Although adrenaline was much higher among semi-continuous group (50\% compared to $23 \%$ among interrupted group); the difference was non significant. GTN was non significantly higher among interrupted group (100\%) compared to semi-continuous one (89\%).

Regarding the postoperative complications, we found that postoperative complications were significantly higher among interrupted group (54\%) compared to only 2 cases $(11 \%)$ among semi-continuous one ( $\mathrm{p}$ value $=0.046$ ).

In Azam et al. 2015 study, they found that incidence of paraprosthetic leakage (PPL) was low in Group II (patients underwent interrupted suture technique) 2(4\%) compared to Group I (patients underwent SC technique) 3(6\%) but this difference was statistically insignificant $(\mathrm{p}=0.64)($ Azamet al., 2015).

As regards the Pulmonary blood pressure immediate postoperatively, we found that immediate postoperative pulmonary blood pressure was slightly higher among semi-continuous group (40 $\mathrm{mmHg}$ ) compared to interrupted one (38.6 $\mathrm{mmHg})$; with non-significant difference $(\mathrm{P}$ value $=$ 0.740 (NS).

Regarding the immediate postoperative Echo findings, we found that all of the immediate postoperative Echo measures were similar between the two groups, with non-significant differences. 
Regarding the immediate postoperative Echo valve findings, we found that all of the cases showed immediate postoperative normally functioning valves, with the exception of trivial PV seen in only 2 of the semi-continuous cases; with nonsignificant difference between the two groups.

Regarding the 1 month postoperative Echo data, we found that after one month of the operation, all of the Echo findings were similar between the two groups, with non significant differences between them.

Regarding the one month postoperative Echo valve findings, we found that after one month, valves were normally functioning among all cases, with the exception of trivial PV, mild PV and central $\mathrm{MR}$; seen in one case of the interrupted group each; with non significant difference between the two groups.

Regarding the 3 month postoperative Echo data, we found that after 3 months of the operation, EF and LVPWT showed non significant differences between the two groups. The exception was PHT which was significantly higher among semicontinuous group compared to the interrupted one; with a significant difference ( $\mathrm{p}$ value $=0.033$ ).

Regarding the three month postoperative Echo valve findings, we found that after three months, valves were normally functioning among all cases, with the exception of trivial PV, mild PV and moderate $\mathrm{PV}$; seen in one case of the semicontinuous group each and mild central MR, seen in one case of the interrupted group; with nonsignificant difference between the two groups ( $p$ value $=0.292(\mathrm{NS})$.

Regarding the associated TR, we found that after three months, the majority of cases had either TR (seen in around $75 \%$ of cases); which was either mild or moderate in most of the cases, and severe only in 2 cases, with non significant difference between the two groups ( $\mathrm{p}$ value $=0.322(\mathrm{NS})$.

\section{Conclusion:}

This study showed that the semi-continuous sutures gave similar outcome to the interrupted sutures, with significantly better operative time regarding total bypass time, cross clamp time and cardiopulmonary bypass time.

\section{References:}

Azam H,NaseemA, Mirza A, R, Sara Z, Syed R A G. (2015). Comparison of semi-continuous and interrupted suture techniques for mitral valve replacement. J Pak Med Assoc, 65 (8).

Bonnefoy E, Girard C, Robin J, Ninet J, Barthelet N, Perinetti M et al., (1995). Systematic transesophageal echocardiography during the postoperative first 24 hours after mitral valve replacement.Arch Mal Coeur Vaiss, 88: 315-319.

Carabello, (2005). Modern Management of Mitral StenosisCirculation, 112(3): 432-437.

Cooley,(1984). Techniques in Cardiac Surgery. 2nd ed. Philadelphia: W.B.Saunders. 201-14.

Genoni, Vogt P, Seifert B, Jenni R, Künzli A, Turina $M$ et al., (2000). Paravalvular leakage after mitral valve replacement: improved long-term survival with aggressive surgery?17 Eur J Cardiothorac Surg., 14-19.

\section{Honda ST, Kawasaki H, Shiraishi M, Yamano T,} Kamitani S, Matoba (2016). Mitral Valve Prolapse Revisited Circulation, 133(6): e380-382.

Ionescu A, Butchart E. (2003).Prevalence and clinical significance of incidental paraprosthetic valvar regurgitation: a prospective study using transoesophageal echocardiography Heart, 89: 1316-1321.

Qicai H, Zhengfu H, Weiming Z, Zhoumiao C, Dingsheng $Y$, Bingtang $Z$ et al., (2006). Continuous?Suture Technique in Aortic Valve Replacement. J Card Surg., 21: 178-181. 